J. G. P. WILLIAMS mentioned a trial he had done comparing Phenylbutazone with Ibuprofen, and in the small numbers studied, young patients free from chronic disease, there seemed to be more subjective side-effects from Ibuprofen than from Phenylbutazone.

D. S. MUCKLE agreed that side-effects in sportsmen are difficult to evaluate; the professional player needs his weekly wage packet and ignores minor ailments, but the amateur, looking to a distant Olympic medal, seems to have an enhanced body image and is easily upset by minor ailments. He had observed negligible side-effects in professional sportsmen on either Ibuprofen or Phenylbutazone.

B. CORRIGAN felt that the role of prostaglandins in soft-tissue injury was not proved, in contrast to its presence in chronic rheumatic diseases.

D. S. MUCKLE mentioned recent reports, given at a meeting of the British Orthopaedic Research Society relating to knee sprains in animals, contusions to muscles in animals and man, and ultra-violet damage to skin all increase prostaglandin production. Small animal experiments suggest that this prostaglandin surge lasts only some twelve hours, after which lbuprofen blockage is not very effective.

Prostaglandins can be of benefit by delaying the immune response, but whether they delay healing is uncertain. They may delay fibrocyte formation, and $\mathrm{F}$ series prostaglandins are implicated in collagen biosynthesis.

M. BUSSON mentioned an unpublished trial with Ibuprofen, Indomethacin and a placebo used in the treatment of acute ankle injuries. The group treated with Indomethacin, the most potent inhibitor of prostaglandin synthetase, responded most rapidly, compared with the placebo group, and the Ibuprofen group were in between.

J. P. S. ENGLAND asked if Ibuprofen given before a match had any beneficial effects on injuries.

D. S. MUCKLE doubted the legality or ethics of such a procedure, even though this drug had not been shown to enhance performance in any way.

J. G. P. WILLIAMS has used prostaglandin inhibitors before manipulations and surgical procedures, but not as a properly designed trial.

P. R. TRAVERS pointed out that the concern the athlete feels over minor disability or malfunction does not imply that he is a hypochondriac; just someone who must keep his body in good condition in order to compete.

\title{
OPEN FORUM
}

P. R. TRAVERS invited comments on the cardiovascular aspects of running, and jogging in particular.

D. S. MUCKLE summed up the problem by defining jogging as a hobby for the unfit. Too many think there is no need for training, and few try to get into condition first. Deaths have occurred in Canada and the USA while jogging, and in the UK as well, usually through ventricular arrhythmias. The value of preliminary medical examination, and of electrocardiography, is still controversial, but training with gradually increasing load before attempting maximum exertion is essential for the middle aged. Stroke volume will increase with training, and the resting pulse rate will decrease. Encouragement may be given by pointing out that, as running increases the bulk of the thigh muscles, it will have a similar effect on the heart.

P. R. TRAVERS recounted experience with hypertensive patients, who were given very carefully graduated exercise, within their own tolerance, and does not consider hypertension per se a contra-indication to exercise. Very few "abnormal" ECGs were noted, and in seven years only one was considered by a cardiologist to be significant.

With veteran classes in sport, many older people who resume training will have a slower resting pulse, which can easily be confused with the pathological bradycardia of the "sick sinus" syndrome. 
J. G. P. WILLIAMS pointed out that pathological conditions likely to develop while running were unlikely to be detected by a traditional medical examination. There is a danger that older people who have passed such an examination will assume that they are fit to do whatever they want. Even a negative cardiological examination is no guarantee against coronary ischaemic death a short time afterwards.

As basic fitness levels vary, it is impossible to prescribe an exact exercise load to suit everyone. Each person must be assessed individually, and his exercise load ascertained by his response to graduated training. Amongst ten thousand patients in a rehabilitation unit where vigorous exercise is the rule, there has only been one death from ischaemic heart disease, and no form of medical examination could have prevented that.

P. RENSTRÖM found no particular cardiac hazard amongst Swedish skiiers and distance runners competing in very cold weather conditions.

P. R. TRAVERS considered education a most important aspect of exercise for older people. The doctor has such an opportunity if he conducts pre-training medical examinations, and the value of exercise in the preservation of mobility, strength and cardiovascular reserve can be stressed. Simple health measures such as walking, dietary control and avoidance of smoking can be encouraged. A full medical examination will reveal muscle wasting, early osteoarthritis and bronchospasm. Jogging should be for enjoyment and not allowed to become competitive, with the attendant risks of over-stressing limbs and circulation.

Work done for the Institute of Directors suggests that any exercise which puts stress on the heart improves coronary circulation; a larger thrombus is therefore needed to cause infarction, and anastomotic vessels reduce the extent of death of muscle fibres.

A. YOUNG raised the point of comparing the effects of short duration vigorous activity such as squash with steady daily jogging.

D. S. MUCKLE and co-workers found that vigorous exercise for twenty minutes two or three times a week maintained biochemical fitness. For an athlete three one-hour heavy training sessions a week are all that is needed to attain competition fitness, and for a recreational participant two twenty minute games of squash, should be sufficient. Many international athletes over-train.

J. G. P. WILLIAMS agreed that this applied to endurance athletes especially, who frequently practice the quantity of training rather than the quality. Many specialised games players recovering from injuries improved their general standards of fitness during supervised rehabilitation, and this led to improved performance in their games. Many players train too specifically, often to the neglect of general fitness training.

P. R. TRAVERS agreed that many young players started specific skill training too soon, before they had developed aerobic and anaerobic capacity, strength and mobility.

J. G. P. WILLIAMS mentioned that most fitness parameters in athletes were higher just before the beginning of the competitive season, and declined as the season advanced. To too many doctors, "fitness" means only "absence of ill health".

\section{CHAIRMAN'S CLOSING REMARKS}

P. R. TRAVERS summing up the forum and the symposium, emphasized the general agreement among the speakers concerning the problems injured sportsmen produce.

$75 \%$ of injuries occur to people below the age of 30 , most of whom are engaged in employment needing fitness, in professional sport, in business, professions and industry. Early treatment is therefore necessary if the period of disability is to be kept to a minimum.

Treatment is only effective if a correct diagnosis has been made, and the mechanism of the injury understood, and possible pathology envisaged. 